\title{
Hydroxy-Chloroquine: Chemoprophylaxis of Malaria and not a Silver Bullet versus COVID-19
}

\author{
Irshad Hussain
}

Institute of Pharmacy, SMBB Medical University Larkana, Pakistan

Article info.

Received: July 14, 2020

Accepted: July 23, 2020

Funding Source: Nil

Conflict of Interest: Nil

Cite this article: Hussain I. Hydroxy-

Chloroquine: Chemoprophylaxis of Malaria

During the Pandamic Covid-19. RADS J

Pharm Pharm Sci. 2020; 8(1):65-67.

*Address of Correspondence Author: irshadpharmacist@yahoo.com

\section{A B S TRACT}

Introduction: The world has been facing the pandemic COVID-19. This article highlights the importance of Hydroxychloroquine (HCQ) and Chloroquine (CQ) in the chemoprophylaxis of malaria during the pandemic COVID-19.

Objective: To derive logical conclusion about the indicated use of $\mathrm{HCQ} / \mathrm{CQ}$ in malaria during the pandemic COVID-19.

Methods: Relevant research articles were retrieved. The online reports, research articles from google, google scholar and PubMed databases were searched and analyzed.

Results: $\mathrm{HCQ}$ and $\mathrm{CQ}$ are indicated for Malaria and its prophylaxis. The drugs are not silver bullets versus the COVID-19. The available clinical data till the end of July 2020 can be sufficient for the health authorities of various countries to stop using HCQ/CQ to treat COVID-19 patients. The drugs are indicated for malaria instead. Malaria, a potentially deadly parasitic disease in the poorest countries which cannot even afford its preventive measures with $\mathrm{HCQ} / \mathrm{CQ}$ and other drugs. Misuse of available drugs of Malaria can worsen the disease in the Africa and some Asian countries. The World Health Organization has recommended continuing chemo-prophylactic efforts for malaria during the COVID-19 crisis. Malaria and COVID-19 have certain common symptoms such as fever, headache and body pain which doubles the risk through misleading diagnosis by considering COVID-19 patients as malaria patients and vice versa. WHO has recommended the mass distribution of antimalarial drugs for these counties, so that malaria could not make COVID-19 more deadly.

Conclusion: Use of $\mathrm{CQ} / \mathrm{HCQ}$ may be continued for the chemoprophylaxis of Malaria during the pandemic COVID-19 and the drugs may not be considered as Silver bullet for COVID-19. Directions of FDA and WHO may be complied regarding use of $\mathrm{CQ} / \mathrm{HCQ}$.

Key words: HCQ, Hydroxychloroquine, $\mathrm{CQ}$, Chloroquine, Chemoprophylaxis of Malaria, COVID-19
This manuscript highlights the need to continue the conventional use of hydroxychloroquine for the chemoprophylaxis of malaria during the COVID-19 pandemic by discussing the current controversies developed with the use of drugs to manage COVID-19 by certain countries based on insufficient clinical information and by the excessive influence of certain government officials those otherwise could not develop an effective strategy to minimize the threats of COVID-19 since 
the alarming outbreak from Wuhan., in China. Malaria, a life-threatening parasitic disease of the poorest countries (mainly the African region), those, that cannot even afford its preventive measures / prophylactic drugs. The African region carries up to $90 \%$ of the global burden of malaria [1]. Causative agent of the disease is plasmodium parasite that is transmitted by female anopheles mosquito (vector of malaria). It affects more to children than adults. Due to malaria, an estimated 0.405 million deaths worldwide were reported in 2018. Symptoms of malaria are fever, chills, headache, anemia, respiratory distress, multiple organ failure and even deaths in the absence of treatment [2]. Se asonal prophylaxis and malaria prevention campaigns, provide the community with a preventive dose of antimalarials such as hydroxychloroquine (HCQ) or chloroquine (CQ) and other medicines if a resistant strain of plasmodium is confirmed. WHO has already recommended preventive measures against malaria. Although advanced research drugs for malaria are also currently available, but quinine derivatives are still used for the prevention and control of malaria [3]. Current pandemic caused by the novel SARS-Cov-2 has wreaked havoc in the lives of people from almost all countries with the main symptoms of hot fever, cough, shortness of breath and sore throat, etc [4]. WHO has recommended continuing chemo preventive efforts for malaria during COVID19. WHO has also closely monitored trials of quinine derivatives for the prevention and treatment of COVID-19 [5]. COVID-19 and malaria have certain common symptoms such as fever, headache and body pain that can double the risk of misleading diagnosis. $\mathrm{WHO}$ has therefore recommended mass distribution of anti-malaria drugs in the poor countries, so that malaria could not make COVID-19 more deadly [6]. Effects of Chloroquine were studied versus

COVID-19 in vitro. [7]. Randomized controlled trials were needed to better assess the clinical efficacy of $\mathrm{CQ} / \mathrm{HCQ}$ in COVID-19 [8]. There is available invitro data related to efficacy of $C Q / H C Q$ against viruses such as HIV and SARS-Cov-1 $[9,10]$. The Indian Ministry of Health had approved the use of $\mathrm{HCQ}$ in the chemoprophylaxis of COVID-19 for asymptomatic healthcare workers in COVID19 and for family members of confirmed cases of COVID-19, the approval with the prescription of a licensed practitioner. The Indian Ministry of Health had ignored the requirements of essential clinical evidences mandatory for such approvals. There were no enough clinical trials to assess the efficacy of the use of CQ/HCQ in COVID-19, as stated by WHO. Several counties in the Middle East had also authorized the use of HCQ in COVID-19 [11, 12]. Since the start of the COVID-19 epidemic in Wuhan, the Chinese had attempted to trial HCQ to minimize the symptoms of COVID-19 patients, and such use has been reported in a multicenter clinical trial in China [13]. Use of $\mathrm{HCQ}$ to minimize symptoms of COVID-19 was also announced by the president of United States. The president was criticized by the relevant international health agencies because advocating the use of $\mathrm{CQ} / \mathrm{HCQ}$ without sufficient clinical information was not scientific. Under such circumstances, the Food and Drug Administration (FDA) announced HCQ's emergency approval for use in serious COVID19 patients in hospital settings and has now restricted use for clinical trials in hospital settings only, although, FDA had revoked this use. Being one of the main producers of $\mathrm{HCQ}$, India had sent donations of HCQ stocks to 30 countries, including SAARC countries (except Pakistan), mentioning the requests for assistance by those counties, particularly in the United States where the death toll was alarming. Surprisingly, India was also pushed by the United States president for consequences in the event of non-supply of $\mathrm{HCQ}$ [14].

In such a scenario of seasonal malaria, COVID-19 pandemic and the risks of co-infections, the author on the basis of recent clinical evidences suggests to support a campaign to administer the CQ / HCQ prevention dose for malaria, among the population at high risk of malaria.

1. Malarial prophylaxis to reduce doubling the risk of misleading diagnosis of considering a malaria patient as a COVID-19 patient. Malarial prophylaxis cannot make COVID-19 more deadly by preventing the possibility of co-infection of COVID-19 and malaria.

2. Reducing the prevalence of malaria and minimizing the crowd of suspects at COVID-19 testing centers, as similar symptoms of fever and headache can increase the concerns of malarial victims to confirm it by reaching test centers thus increasing the probability that only COVID-19 patients will reach the 
test centers and hence preventing the wastage of test kits and other resources.

FDA has revoked the emergency use authorization (EUA) of $\mathrm{CQ} / \mathrm{HCQ}$ in the COVID-19. There are serious safety issues with the use of these drugs mainly heart rhythm problems.. The drugs having no benefit in decreasing the probability of death rate and propelling the recovery [15]. WHO has also stopped the use of $\mathrm{CQ} / \mathrm{HCQ}$ in international clinical trial of WHO based on the interim results of drug use as compared to the standard of care. [16]. In such circumstances the use of $\mathrm{CQ} / \mathrm{HCQ}$ may be continued in approved indications especially the prophylaxis of Malaria during the pandemic COVID-19

\section{REFERENCES}

1. Breman, JG., et al. 2001. The Intolerable Burden of Malaria: A New Look at the Numbers. Journal of Tropical Medicine and Hygiene. American Society of Tropical Medicine and Hygiene. 64(1), https://www.ncbi.nlm.nih.gov/books/NBK2622/.

2. https://www.who.int/news-room/factsheets/detail/malaria (accessed 14 April 2020).

3. Steffen, R., et al. 1990. Malaria chemoprophylaxis among European tourists in tropical Africa: use, adverse reactions, and efficacy. Bull World Health Organ. 68(3), 313-322.

4. http://covid.gov.pk/ Date Accessed 15 April 2020.

5. https://www.who.int/news-room/q-a-detail/malariaand-the-covid-19-pandemic. (Accessed 15 April 2020).

6. http://www.rfi.fr/en/africa/20200411-covid-19-willmake-malaria-tracing-and-treatment-much-harderwho (Accessed 15 April 2020).

7. Jia Liu., et al. 2020. Hydroxychloroquine, a less toxic derivative of chloroquine, is effective in inhibiting SARS-CoV-2 infection in vitro. Cell Discovery. 6;16. https://doi.org/10.1038/s41421020-0156-0.
8. Kerstin Frie., Kome Gbinigie., 2020. Chloroquine and hydroxychloroquine: Current evidence for their effectiveness in treating COVID-19. CEBM. https://www.cebm.net/covid-19/chloroquine-andhydroxychloroquine-current-evidence-for-theireffectiveness-in-treating-covid-19/.

9. Chauhan, A., Tikoo, A., 2015. The enigma of the clandestine association between chloroquine and HIV-1 infection. HIV medicine. 16(10), 585-590.

10. Vincent., et al.. 2005. Chloroquine is a potent inhibitor of SARS coronavirus infection and spread. Virology journal,. 2(1). 69.

11. https://www.bbc.com/news/51980731. (Accessed 17 April 2020).

12. Secretary (Health and family welfare). Advisory on the use of Hydroxy-chloroquine as prophylaxis for SARS-CoV-2 infection. March 2020. Ministry of Health and family welfare, Nirman Bhawan, New Delhi-110008.

13. Jianjun Gao., et al., 2020. Chloroquine phosphate has shown apparent efficacy in treatment of COVID-19 associated pneumonia in clinical studies. Biosce Trends. 16;14(1):72-73. DOI: 10.5582/bst.2020.01047.

14. https://www.washingtonpost.com/business/2020/0 4/06/faq-chloroquine-hydroxychloroquinecoronavirus/?utm_campaign=wp_politics_am\&utm _medium $=$ email\&utm_source $=$ newsletter\&wpisrc $=$ nl_politics. (Accessed 15 April 2020).

15. http://www.fda.gov/drugs/drug safety-andavailability/fda-cautions-against-usehydroxychloroquine-or-chloroquine-COVID-19 outside hospital setting-or (Date accessed 30 July 2020).

16. https://www.who.int/news-room/detail/04-07-2020who-discontinues hydroxychloroquine-andlopinavir-ritonavir-treatment-arms-for-COVID-19 (Date accessed 30 July 2020. 